\title{
A Preliminary Exploration on Internet of Intelligences Based on Cloud Computing
}

\author{
Yannan Sun ${ }^{1,2,3}$ Chongfu Huang ${ }^{1,2,3, *}$
}

${ }^{1}$ Key Laboratory of Environmental Change and Natural Disaster, Ministry of Education of China, Beijing Normal University, Beijing 100875, China

${ }^{2}$ State Key Laboratory of Earth Surface Processes and Resource Ecology (Beijing Normal University), Beijing 100875, China

${ }^{3}$ Academy of Disaster Reduction and Emergency Management, Ministry of Civil Affairs \& Ministry of Education, the Peoples' Republic of China, Beijing 100875, China

\section{基于云计算的智联网初探}

\author{
孙雁南 ${ }^{1,2,3}$ 黄崇福 $1,2,3, *$ \\ '北京师范大学环境演变与自然灾害教育部重点实验室, 北京 100875 , 中国 \\ ${ }^{2}$ 地表过程与资源生态国家重点实验室 (北京师范大学), 北京 100875 , 中国 \\ ${ }^{3}$ 民政部/教育部减灾与应急管理研究院, 北京 100875 , 中国
}

\begin{abstract}
In order to use advantage of cloud computing (CC) to improve Internet of intelligences (IOI), in this paper we suggest the concept of Internet of intelligences based on $\mathrm{CC}$, and preliminarily discuss automatic configuration management of information resources in IOI. A sharp distinction between the current IOI and the IOI based on CCis that the improved IOI would have higher efficiency. It is useful for IOI to process vast amounts of information.
\end{abstract}

Keywords: Internet of intelligences, cloud computing, distributed, parallel type

\section{摘要}

为发挥云计算的优势改进智联网, 本文提出了

基金项目: 国家自然科学基金项目（编号 41471424); 国家重大科学研究计划“全球变化与环 境风险演变过程与综合评估模型” (编号: 2012CB955402)。

*通讯作者：黄崇福, hchongfu@bnu.edu.cn
基于云模型的智联网概念, 并对智联网中信息 资源的自动配置管理做了初步探讨。与现行智 联网最大的不同是, 基于云计算的智联网运行 效率更高, 便于智联网处理海量信息。

关键字：智联网, 云计算, 分布式, 并行式

1. 引言

智联网以互联网为依托, 连接可提供有效 信息的智能体, 集万千小智慧为大智慧, 利用 人脑智慧经验的超智能性来解决一些复杂的 问题。黄崇福 (2012) 提出应用智联网解决传 统风险分析中样本信息获取困难、有效信息获 取数量难以保证、风险变化难以捕捉、风险分 析结果可靠性无法验证等诸多题 ${ }^{[1]}$; C.F.Huang (2013) ${ }^{[2]}$,艾福利 (2013) ${ }^{[3]}$ 提出复式智联网 （智能体不但提供经验信息, 还参与信息的处 理操作) 以及处理柔性信息一种解决思路,智 能体参与经验信息的处理避免了传统计算机 智能算法语言识别易歧义、情景理解困难等短 板问题, 是解决智联网多源异构信息的有效思 路; Zeng $(2013)^{[4]}$ 探讨了智联网对干扰信息的 
Risk Analysis and Crisis Response in Big Data Era (RAC-16)

甄别以及对激励机制的建立等问题，降低恶意 虚假信息的干扰、提高有效信息的利用率的同 时激励智能体的积极性, 为智联网达到有效收 集海量可借鉴智慧经验信息的目的提供了可 行的解决思路; $\mathrm{Wu}$ 等 ${ }^{\left[{ }^{[}\right]}$将智联网与风险雷达相 结合, 初步可实现对风险提前感知、实时监控 的效果。以上对智联网的研究, 已经解决了智 联网发展中的柔性信息处理、干扰信息甄别、 激励机制建立等关键问题, 是智联网平台有效 运行的基础。但是, 智联网作为一个收集海量 智慧信息的智能触角, 如何解决极高工作量对 系统服务器带来的巨大压力, 以及如何合理高 效的利用数据库资源, 做到当某些智慧信息资 源过热时, 系统能自动、及时反应并做出一定 的调整措施, 从而得到智联网大数据资源合理 配置, 实现对海量数据的有效管理也是智联网 平台优化运行效率, 增加优越性以及可持续性 急需要解决的难题。

云计算作为新一代 IT 模式, 其核心理念 是以服务的形式为用户基础架构、平台、软件 等, 整合大规模可扩展的计算、存储、数据、 应用等分布式计算资源, 用户在需要时进行使 用和购买, 依靠其后端规模庞大、自动化和高 可靠性的云计算中心的支持, 用户只要接入互 联网, 不用进行繁琐的安装和维护工作, 就能 访问各种基于云的应用和信息。云计算作为一 个强大的“云”网络, 连接了大量并发的网络计 算机和服务器, 从而将各自的资源通过云计算 平台整合起来, 并虚拟化技术扩展每一个服务 器的能力, 提供超级计算和存储能力。云计算 GFS-Goole ${ }^{[6]}$ 的可扩展的分布式文件系统 (Google File System), 用于对大型的、分布式 的、海量数据的访问应用; 基于Hadoop的开源 数据工具 BT( Big Table) ${ }^{[7]}$ 数据管理技术以及 开源数据管理模块 HBase 和 Hive, 用于存储 和处理海量结构化数据; 云计算虚拟化技术 ${ }^{[8]}$ 隔离软件应用与底层硬件, 每个应用功能的实 现不依赖与其物理平台, 通过虚拟平台进行管 理、扩展、迁移、备份。云计算强大的信息存 储、计算、管理能力, 为解决智联网平台现有 难题, 提供了新的有价值的思路。本文提出将 云计算应用于智联网平台, 对应用云计算的智 联网的优势、基本框架、资管理模式的实现做 出初步探讨。

\section{2. 智联网思想}

2.1 智联网思想的提出

文献[9]第一次给出了智联网的完整思想以

及定义: 设 $A$ 是一个智能体集合, $N$ 是 $A$ 使 用的一个网络, $M$ 是处理 $A$ 所提供信息的模 型, 三元体 $(A, N, M)$ 称为一个智联网, 记 为 $\Phi$ 。

根据智联网的定义, 智能体所使用的网络 $N$ 、智能体 $A$ 和分析处理模型 $M$ 是组成一个完 整智联网的有机整体, 其中, 智能体指具有观 察、演绎、推理和解决问题能力的个体, 是智 联网智慧的来源, 也是需求和信息的来源, 提 供的信息大多是柔性信息, 不仅具有弹性而且 具有伸缩和舞动的空间。智联网利用互联网对 智能体提供的见闻、经验、知识、数据和判断 等海量信息, 利用一定的数学模型进行分析处 理并以合理的方式展现分析结果, 给出总结性 解答。

在已有风险分析、信息处理、互联网技术 等研究的基础上形成的智联网思想, 旨在解决 风险分析中的样本信息获取困难、有效信息获 取数量难以保证、风险分析结果可靠性无法验 证等诸多问题。互联网的高速发展以及巨大用 户群以及需求群, 为智联网的实现提供了重要 的前提依托。智联网在线风险分析, 将信息技 术视为自然灾害风险分析的有效工具, 以强大 的互联网技术为依托, 实现在线实时的动态风 险分析。因此, 智联网的服务流程, 包含需求 表述、信息提供、信息提取、信息分析、信息 分享等步骤 (如图 1 所示)。

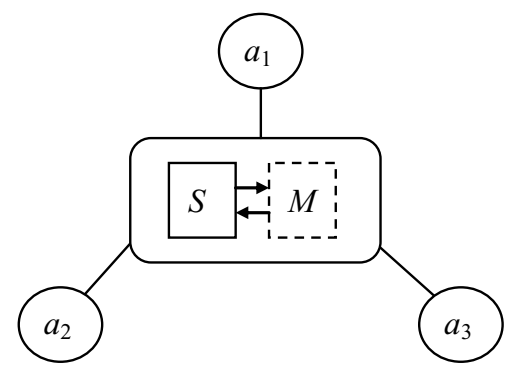

图 1: 最简单的智联网拓扑图, 它由一个网络 服务器 $\mathrm{S}$, 一个数学模型 $\mathrm{M}$ 和三个智能体 $a_{1}, a_{2}, a_{3}$ 组成。 
Risk Analysis and Crisis Response in Big Data Era (RAC-16)

\section{2 智联网拟解决的问题}

智联网思想提出的最初目的, 主要是为了 解决如何在风险分析中有质量有数量的获取 样本信息的问题。互联网的发展以及各种网络 平台、APP 应用市场的成功为智联网平台的实 现提供了可能。以互联网为支撑的智联网, 可 更高效地收集智慧信息, 也可具有更有效的分 析数据信息, 智联网还可通过柔性感知、智联 万千、实时动态、虚拟现实等方法和技术缓解 概率风险分析面临的假设统计规律难以充分 证实、足够多的数据资料支持难以获得、变化 风险变化捉摸不定, 分析结果的可靠性难以保 证等问题。

因此, 智联网提供了一个可以为供需双方 进行智慧服务的平台, 其中, 包括需求者表述 个性化需求, 经验者提供个体经验, 分析模型 进行信息处理、分析、分享等一系列流程, 从 而实现风险问题的智慧服务。因风险问题类型 的不同, 风险分析的流程必然存在很大差异, 这同时也决定了不同的智联网类型, 如地震、 洪水等自然灾害风险问题可定位为监测型智 联网, 以收集经验者的异常信息以及经验判断 为主; 气象灾害、地质灾害等较为致灾因子、 㝋灾环境较为复杂的风险事件的风险分析可 定位为综合型智联网。虽然不同类型智联网主 要针对不同的风险事件, 但风险问题分析中的 核心过程是相同的, 即信息的收集与信息的分 析。

\section{3.云计算}

美国国家标准与技术研究院定义云计算 为: 一种用户可按需通过网络访问可配置计算 机资源池 (网络、服务器、存储设备、应用程 序以及服务等) 的模型, 并且提供方只需参与 最少的管理或服务, 即可快速享受资源供应服 务以及资源发布 ${ }^{[10]}$ 。计算机资源服务化是云计 算重要的表现形式, 核心思想旨在为用户屏蔽 数据中心管理、大规模数据处理、应用程序部 署等问题, 通过云计算, 用户可根据其业务负 载快速申请或释放资源, 并以按需支付的方式 对所使用的资源付费, 在提高服务质量的同时 降低运行维护成本。

云计算的特征主要有: 自服务 (用户自己 申请需要多少资源、什么样的资源)、虚拟化、
高效和灵活、按需付费、统一的计算模式; 其 部署模型有: 私有云、社区云、公有云、混合 云等多种形式; 其服务类型分为: 基础架构即 服务 (IaaS), I IaaS 提供硬件基础设施部署服 务, 按照用户的需求提供实体或虚拟的计算、 存储和网络等资源、平台即服务 $(\mathrm{PaaS}), \mathrm{Paa}$ $\mathrm{S}$ 是云计算应用程序运行环境, 提供应用程序 部署与管理服务、软件即服务 $(\mathrm{SaaS}), \mathrm{Saa} S$ 是基于云计算基础平台所开发的应用程序。

云计算的核心思想：是实现“计算、存储、 网络”及“软件平台”与“应用”的规模化的管 理, 实现所有资源的整合、分配、调配、调度。 用户无需知道云计算的“云”里包含什么设备 和软件、软件怎样部署、设备怎么连接、能实 现什么样的功能、怎么进行管理等问题, 云计 算会将虚拟化的各种服务集合体打包提供给 用户。

\section{4. 应用于云环境下智联网的优势}

4.1 分散存储和并行计算

基于云计算的模式可以进行分布式并行 信息存储与计算, 可根据智能体提供信息的特 点, 设置不同规模、不同组织形式的计算云, 不同的计算云之间再通过各种相关关系连接 起来, 实现实时调用, 自由组合, 从而提高了 智联网平台的信息存储效率, 大大节省了数据 存储空间, 待需要计算时, 再从各种云调用有 效信息, 提高了信息间的交互程度和计算效 率。

分布式设计可将大规模任务分割成许多 小规模任务, 再将细分后的小任务分散并行处 理, 最后将所有小任务产生的结果归约后作为 初始大任务的最终结果。简单地说分布式系统 遵循: 大任务一任务分割一分散并行处理一归 约分结果一输出最终结果这一抽象流程, 在进 行大数据存储、处理、分析上具有得天独厚的 优势。

例如, 利用智联网服务平台收集地震宏观 异常信息, 评估地震风险时, 若将全国范围按 照一定规则划分为不同区域, 并为每个区域设 置私有云 (只允许本区域的相关人员增添改评 其数据库信息, 其他区域相关人员只能查看或 调用其数据库信息), 这样智联网平台既可以 在私有云规模上评估私有云覆盖区域的地震 


\section{Risk Analysis and Crisis Response in Big Data Era (RAC-16)}

风险, 也可以通过不同地区私有云的调用与叠 加等操作, 评估更大区域范围内的地震风险, 同时, 因为数据库信息之间的关联是通过调用 实现的, 从而避免了信息的重复录入, 节省了 数据库的存储空间, 提高了数据库信息的使用 效率。

\section{2 开放的服务环境}

传统的平台一般都只有一到两个终端服务 器支撑, 而云计算模式中的 “云” 是由百万台服 务器组成的服务器集群, 又由于云计算环境是 完全开放的服务环境, 它的体系结构、标准、 系统平台、软件服务等都是开放的, 并且这些 服务分布在各地数以万计的不同服务器上, 它 们之间的彼此关联和作用构成了多种不同的 模式, 使得智联网服务平台的数据信息规模大 大增加, 为智联网收集计算的海量智慧信息的 实现提供了可能。

“云”的规模可控性强, 云计算支持用户在 任意位置使用各种终端获取应用服务, 用户所 请求的资源来自 “云”, 大大解放了系统服务器 的压力; 云计算可以支持数据多副本容错、计 算节点同构互换等多种保障服务, 性能上比本 地计算机更加可靠; 通用性强, 支持多个不同 类型的应用同时运行, 可满足不同程度的应用 扩展, 以及容纳大量用户的高扩展性; 用户可 按照自身的需求按需购买服务, 大大节省用户 前期软硬件投入投入, 低成本高自动化的服务 可使得智联网服务平台拥有巨大的潜力以及 吸引用户的能力; 系统的维护工作可交给“云” 另一端最专业团队, 降低维护成本, 优化维护 过程。

\section{3 多源的服务模式}

面向不同的客户群体, 云计算环境可扩展 出不同的服务模式, 有利于智联网服务平台根 据不同的用户需求特点定制不同的服务模式, 云环境下的智联网平台的呈现多源化的服务 模式, 为用户需要的任何其他应用软件, 都可 以通过一定的方式与智联网服务平台整合的 实现提供了可能。

例如, Sales-force公司在其 sforce 客户/ 服务整合平台上提供按需服务的客户关系管 理, 允许客户与独立软件供应商定制并整合其
产品, 进而建立他们各自所需的应用软件; Google App Engine平台能够让用户在 Google 的基础架构上运行自身的应用程序, 并可根据 用户的访问量和数据存储需求实施动态扩展; 而 Amazon 允许用户对其所用资源付费的用 户，通过 Amazon Web Service程序访问 Amazon 存储、计算、消息传递和数据集等的 计算基础设施 ${ }^{[11]}$ 。

\section{5. 基于云计算的智联网服务平台框架}

(1) 框架概念模型

基于云计算的智联网服务平台的基本概 念框架是通过智联网平台收集智能体智慧信 息传送到数据库, 再由云计算平台对数据库信 息进行管理、处理、计算、等业务操作, 客户 再通过应用接口访问信息处理结果等, 如图 2。

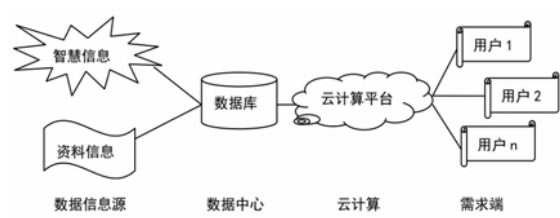

图 2, 基于云计算智联网服务平台概念模型

（2）总体框架模型

基于云计算的智联网服务平台框架, 自底 向上分别为智联网、智慧数据库、云计算平台、 用户界面等, 理论上来说这是一套从数据生成 到组织、存储、查询、分析、服务完整的框架 性体系结构, 为针对用户需求量身定制的智慧 囊。

用户作为信息需求者, 可通过普通浏览 器、客户端应用程序、平板电脑、手机等各类 终端访问智联网平台, 发布自身需求 (希望通 过智联网解决的问题); 以智联网作为智慧信 息采集的触角, 实时收集不同智能体对用户提 问事件的经验、看法、解决办法等第一手资料, 智联网平台通过网络将用各种方式采集或人 为加工的数据信息统一存储到数据库中心, 基 于云计算搭建的云计算平台, 采用虚拟化等系 列核心技术, 实现数据云存储、云计算, 在专 用网络中建立私有云、Internet 上建立公有云, 并提供相应的云服务 (不同粒度的数据服务、 
计算服务等), 以进行软硬件资源管理、空间 聚类分析、可靠性计算、实现潜力分析等; 并 查看云端数据信息分析处理结果, 如图 3。

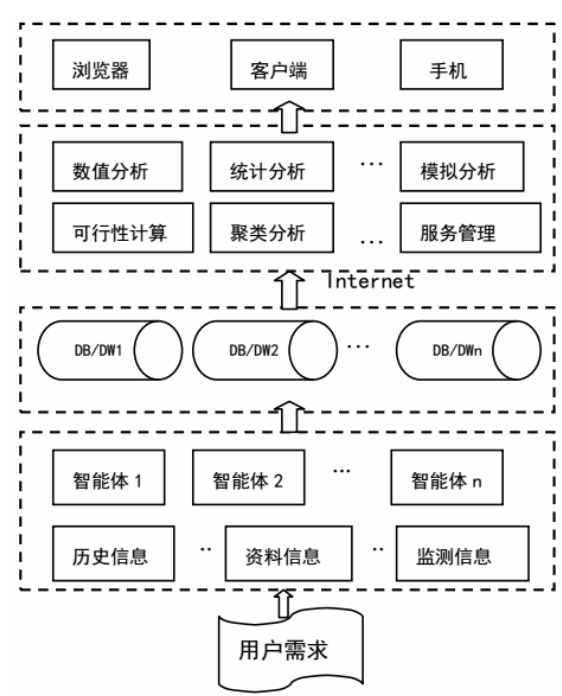

图 3, 基于云计算的智联网平台框架体系结构

\section{6. 分布式存储计算的实现关键性技术支持}

Google appengine 提供在云环境下的程序应 用平台的开发、运行与部署, 其对数据的分布 式并行式存储、编程模式的技术基础, 为实现 能够分布式存储计算数据信息的智联网平台 提供上了技术上的可能。

\section{1 分布式文件系统}

大型的、可扩展的分布式文件系统Google file system ${ }^{[12]}$, 重要针对超大数据的存储与处 理, 解决云计算数据迅速增长问题。Client(用 于程序访问接口)访问GFS时, 首先访问Master (主服务器, GFS管理节点), 获取与节点交 互的chunk server（数据块服务器） 信息, 完 成数据存取工作。Client与chunk server之间跳 过与Master的数据交互过程, 直接进项数据信 息传输, 并行式访问则在交互的数据被分成多 个数据块分布式存储时实现。GFS设计在降低 Master负载的同时, 使得整个系统的输入输出 可并行式处理; 采用中心服务器管理模式, 主 服务掌握系统中所有数据块服务器的情况, 更 新数据块只需要在Master上注册即可, 避免了 在每个chunk server上进行大量重复增添改查 工作，大大增加了系统的可扩展性。

\section{2 分布式计算编程模型}

痛 Mapreduce作为一个软甲构架, 可用 java、 $\mathrm{c}++$ 等语言对海量数据进行并行编程模式处理 盛。借鉴了函数式和矢量编程语言的思想的优 䞇, 适合用于非结构化和结构化的海量数据的 践索、挖掘、分析与机器智能学习等; 编写的 应用程序能够在大型服务器集群上运用, 可对 霰据进行 TB级别的可靠容错并行处理; 计 玨节点和存储节点同点放置的配置使得在已 得好数据的节点上能够高效进行调用、计算、 调试等操作, 避免了数据在网络上的海量传 椾,

平 Mapreduce 的核心思想就是将一个复杂运 員问题拆分成大量 Map 和 Reduce 任务, 在 Map 阶段读取输入文件块, 进行并行分析处 理, 并将处理后的中间结果保存在 Map 任务 执行节点; 在 Reduce 阶段, Reduce 任务读 取并合并多个 Map 任务的中间结果。用户只 需提供自己的 Map 函数以及 Reduce 函数就可 以在云端进行大规模的分布式数据处理。

例如, 统计社交网络中的热点话题时, 可 将全部页面数据存入 GFS, 然后 MapReduce 编 程模式将所有页面数据分配给万千台 Map 服 务器进行计算, Reduce 服务器汇整 MAP 中间 数据, 并生成热点话题排行、热度等需要的结 果信息。

\section{3 分布式数据存储系统}

Bigtable作为放置各种分布式资料的数据 库, 其存储格式是一个有序的分布式多维映射 表, 用来将数据存储或部署到各个计算机节点 上, 实现存储结构的优化 ${ }^{[14]}$ 。可通过一个行关 键字、列关键字、时间䧳进行索引, 配合 MapReduce构架进行对海量数据库复杂的分 析和查询功能。

\section{7.云环境下智联网平台的自适应管理模型}

随着云计算中心信息规模越发庞大和复 杂, 其数万计结点的规模无疑会给云信息资源 维护带来极大的困难。要解决云计算中心数据 信息的的复杂性、大规模性和动态性, 减少在 


\section{Risk Analysis and Crisis Response in Big Data Era (RAC-16)}

云环境下智联网平台的管理中人工操作, 必须 解决如何让云环境下的智联网系统可根据配 置策略自行管理问题, 从而达到云资源的自适 应管理。本文尝试研究云环境下智联网系统的 自适应管理模型的一个框架图, 如图 4。

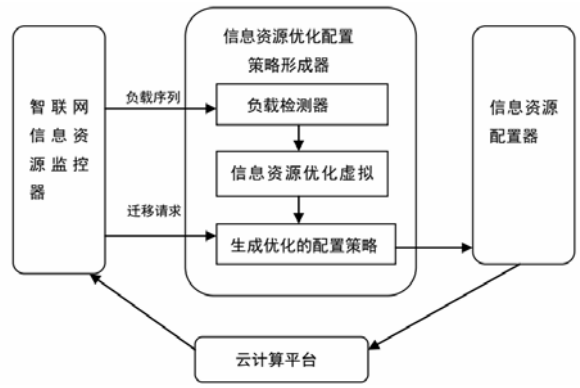

图 4: 基于云计算的智联网平台自管理模型

云计算中所有的资源都是以虚拟机为资 源管理的基本单位, 以一定的方式或者规则放 置在不同的物理机中, 也就是说, 云计算平台 相当于一个提供各种信息资源的屋子, 物理机 相当于屋子里大小不一的盒子, 虚拟机则相当 于大小形状不同、功能各异的物品。云计算中 心通过对虚拟机的监控、分配、迁移和回收实 现对虚拟资源的统一管理, 也就是要考虑怎样 放置盒子里的物品, 才能在得盒子使用数量最 少的情况下达到物品位置的变动最少。那么, 要实现云中心信息资源管理的自适应管理, 需 要有一套优化虚拟机配置方案, 使得需要迁移 的虚拟机可通过优化方案找到新的物理机进 行迁移, 从而实现自动配置管理。第一, 判定 一个物理机是否过载, 过载时需要选择一个或 多个其他虚拟机使得信息资源从该过载物理 机上迁移出去; 第二, 判定一个物理机是否低 载, 低载时需要把所有虚拟机从该物理机上迁 移出去; 第三, 根据虚拟机配置策略为需要迁 移的虚拟机找到新的合适的物理机。

基于云计算智联网平台信息资源自适应 管理模型主要包括 3 个功能模块, 分别是: 智联网信息资源监控器、信息资源优化配置策 略形成器、信息资源配置器。智联网信息资源 监控器实时监控云计算平台各个资源的使用 情况, 并记录到日志; 信息资源优化配置策略 形成器根据一定的规则或者算法生成优化的
虚拟机配置方案, 其中的负载检测器检测虚拟 机的负载情况, 对于超载的虚拟机发出迁移请 求, 然后信息资源优化虚拟器根据虚拟机发出 的迁移请求, 根据一定的算法或者规则寻找优 化的虚拟机配置策略, 从而得到适合超载虚拟 机迁移的新的供体虚拟机, 其配置策略优化目 标是, 使用物理机个数使用较少的同时减少使 虚拟机迁移次数; 信息资源配置器根据信息资 源优化虚拟器的配置策略进行虚拟机迁移调 整。

\section{8. 结论}

云计算大规模并发式连接基础构架、平 台、应用等, 整合可扩展的各种计算资源, 提 供强大的计算存储能力。本文将云计算虚拟 化、海量数据管理等关键技术思想应用在智联 网平台中, (1) 可提高智联网平台的信息存储 效率, 节省了存储空间, 有需要是调用云资源, 提高了信息间的交互程度和计算效率; (2) 完 全开放的服务环境, 关联数万分布在各地的服 务器, 可为智联网海量智慧信息资源的收集提 供必要的基础条件; (3) 所有用户请求资源都 放在基础设置良好的云端, 可解放智联网服务 器压力; (4)云计算超强可扩展性, 可扩展出不 同的服务模式, 有利于智联网服务平台根据不 同的用户需求特点定制不同的服务模式。通过 探讨应用云计算的智联网的诸多优势, 为解决 智联网高效合理利用数据库智慧信息资源, 对 信息资源的合理配置、管理作出及时动态的反 应提供一种可行的解决思路。

\section{参考文献}

[1] 黄崇福, 风险分析的困惑和智联网的使命. 风险分析和危机反应的创新理论和方法, 黄崇福, 翟国方编. 巴黎: Atlantis 出版社, pp.1-6, 2012.

[2] C.F. Huang. The measurement of effective knowledge in multiple Internet of intelligences and application in risk assessment. Intelligent Systems and Decision Making for Risk Analysis and Crisis Response, Eds. C.F. Huang, C. Kahraman, Boca Raton, USA: CRC Press, pp. 1-8, 2013. [3]艾福利. 自然灾害风险分析智联网服务平台 
Risk Analysis and Crisis Response in Big Data Era (RAC-16)

构建与应用研究.北京师范大学博士学位论 文, 2013.

[4] F.L. Zeng, C.F. Huang, E.L. Ai. operation mechanism and interfered information's screening strategy of internet of intelligence. Intelligent Systems and Decision Making for Risk Analysis and Crisis Response, Eds. C.F. Huang, C. Kahraman, Boca Raton, USA: CRC Press, pp. 313-318,2013.

[5] T. Wu, C.F. Huang F.L. Ai. A discussion on using internet of intelligences to improve risk radar. Intelligent Systems and Decision Making for Risk Analysis and Crisis Response, Eds. C.F. Huang, C. Kahraman, Boca Raton, USA: CRC Press, pp. 831-837,2013

[6] 方巍, 文学志, 潘吴斌, 薛胜军.云计算: 概 念、技术及应用研究综述. 南京信息工程 大学学报(自然科学版)), 2012, 4(4): 351-361

[7] 刘鹏. 云计算技术基础. 2 版. 北京: 电 子工业出版社, 2011

[8] Kamoun F. Virtualizing the datacenter without compromising server performance. ACM Ubiquity 2009,200

[9] C.F. Huang. Internet of intelligences in risk analysis for online service. Journal of Risk
Analysis and Crisis Response, 2011, 2(1): 110-117.

[10] P. Mell, T. Grance. The NIST Definition of Cloud Computing. National Institute of Standards and Technology, 2011.

[11] 杨善林. 基于云计算的多源信息服务系 统研究综述.管理科学学报. 2012, 15(5): 83-96.

[12] S. Ghemawat, H. Gobioff, S.T. Leung. The Google file system. ACM SIGOPS Operating System Review. ACM, 2003, 37(5): 29-43.

[13]J. Dean, S. Ghemawat. Mapreduce: simplified data processing on large clusters. Communication of the ACM, 2008, 51(1): 107-113

[14]F. Chang, J. Dean, S. Ghemawat. Bigtable: A distributed storage system for structured data. ACM Transaction on Computer System(TOCS), 2008,26(2): 4 